\title{
Análise da Produção de Narrativas Digitais no Ensino Superior em Saúde
}

\section{Analysis of the Production of Digital Storytelling in Higher Education in Health}

Maria Augusta Vasconcelos Palácio, Miriam Struchiner *2

\begin{abstract}
${ }^{1}$ Pesquisador, Laboratório de Tecnologias Cognitivas/Núcleo de Tecnologia Educacional para a Saúde(NUTES)/Universidade Federal do Rio de Janeiro (UFRJ). Centro de Ciências da Saúde, Bloco L - Sala 55, Cidade Universitária, Ilha do Fundão - Rio de Janeiro- RJ - Brasil. augustapalacio@yahoo.com.br

\footnotetext{
2 Professor pesquisador, Laboratório de Tecnologias Cognitivas/Núcleo de Tecnologia Educacional para a Saúde(NUTES)/Universidade Federal do Rio de Janeiro (UFRJ). Centro de Ciências da Saúde, Bloco L - Sala 55, Cidade Universitária, Ilha do Fundão - Rio de Janeiro- RJ - Brasil. miriamstru@gmail.com
}

\section{Resumo}

A narrativa digital envolve o potencial da narrativa de facilitar a construção de sentido e o compartilhamento de experiências com as linguagens das tecnologias digitais de informação e comunicação. Ela pode promover a participação ativa e reflexiva dos sujeitos educandos. No ensino superior em saúde, defende-se uma mudança nas práticas pedagógicas tradicionais com a valorização das experiências dos alunos. Na formação em Atenção Primária à Saúde há a necessidade de aproximá-los do cotidiano familiar e social dos usuários do serviço e promover a discussão sobre a relevância dessa formação. $\mathrm{O}$ objetivo do artigo é analisar narrativas digitais produzidas em um blog quanto às linguagens midiáticas utilizadas. Trata-se de uma pesquisa qualitativa descritiva, cujo contexto é uma disciplina de um curso de Medicina. Para a produção das narrativas, os alunos criaram blogs em um ambiente virtual de aprendizagem. Os resultados mostraram a produção de narrativas em multimídia a partir da utilização de outras linguagens midiáticas, como imagens/fotos e vídeos. A análise da narrativa revelou que o uso de diferentes linguagens para apresentar as experiências e a forma como o blog foi explorado são aspectos que sugerem o desenvolvimento de habilidades, o estímulo à criatividade e a reflexão do aluno nesse processo de ensino-aprendizagem.

Palavras-chave: Narrativas digitais, Tecnologias de informação e comunicação, Ensino superior, Ensino da Saúde. 


\title{
Analysis of the Production of Digital Storytelling in Higher Education in Health
}

\begin{abstract}
Digital storytelling involves the narrative's potential to facilitate the construction of meaning and sharing experiences, with the languages of Digital Information and Communication Technologies. It can promote active and reflective participation of the students. In Higher Education in the health field, the latest debates argue for change in traditional teaching practices with the appreciation of the student's experiences. On the training in Primary Health Care there is the need to approach them from the family daily routine and social service users and promote discussion on the relevance of training in that context. The aim of this study is to analyze digital storytelling produced in a blog according to the media languages used. This is a descriptive qualitative research which the context is is a discipline present in a medicine course. To produce digital storytelling, students created blogs in the virtual learning environment. The results showed the production of multimedia narratives based on the use of different media, such as pictures/photos, and videos. Analysis of the narratives revealed that the use of different languages to present the experiences and the way the blog tool was explored are aspects that suggest the development of skills, stimulating creativity, and reflection of the student in the teachinglearning process.
\end{abstract}

Keywords: Digital storytelling, Information and communication technologies, Higher education, Health education.

\section{Introdução}

Este artigo apresenta um recorte da tese de doutorado intitulada Formas de expressão, reflexões e aprendizagens nas narrativas digitais no processo de formação em Atenção Primária à Saúde: um estudo de caso com alunos de medicina, desenvolvida no Programa de Pós-Graduação em Educação em Ciências e Saúde, da Universidade Federal do Rio de Janeiro (UFRJ).

O ato de narrar acompanha todas as nossas ações e relações sociais; ele nos ajuda a dar sentido aos acontecimentos, uma vez que ao narrarmos uma experiência somos remetidos ao registro da memória sobre o cotidiano da vida social, ao específico do sujeito, ao coletivo de um grupo, aos significados que os sujeitos atribuem aos acontecimentos (Almeida \& Valente, 2012). As pessoas contam histórias para dar sentido à experiência e disseminar crenças e valores para os outros. Contar histórias está na base da experiência interpessoal quotidiana e fornece um meio de comunicação, interação, organização, percepção, reflexão, pensamento e, finalmente, ação (Boase, 2013).

Bruner $(1991$; 1996) descreve a narrativa como um modo de pensar, como uma forma de organização da nossa experiência e, portanto, como um recurso no processo da educação. A narrativa está relacionada à produção de sentido e à compreensão sobre como a realidade é construída, considerando a subjetividade, o ambiente cultural e seus instrumentos para o discurso comunicativo. Para o autor, a narrativa assume algumas características que orientam a sua construção e compreensão; dentre elas está a sua organização em início, meio e fim; as narrativas estão relacionadas a aspectos particulares, ou seja, contam experiências individuais, mas sua função reside no fato de permitir o compartilhamento dessa 
experiência; todas as ações têm razões implicadas e são motivadas por crenças, desejos, teorias e valores dos sujeitos; por fim, toda narrativa possui múltiplos significados, pois não há uma interpretação singular (Bruner, 1996).

A narrativa tem ganhado novos significados e usos com o advento das tecnologias digitais de informação e comunicação (TDIC), possibilitando novas perspectivas de trabalho em contextos educacionais. Apresentadas, geralmente, na forma oral ou escrita, com a contribuição das mídias, as narrativas podem ser produzidas digitalmente (Almeida \& Valente, 2012). Caracterizadas como narrativa digital, digital storytelling ou relatos digitales, essas novas formas de representação da narrativa, assim como as tradicionais, guardam as mesmas caraterísticas de se constituírem a partir de um ponto de vista particular do indivíduo ou se relacionarem à apresentação sobre um tema em específico (Robin, 2005; 2008).

Desde o final da década de 1980, o Center for Digital Storytelling - CDS, uma organização sem fins lucrativos em Berkeley, Califórnia, tem fornecido treinamento e assistência para pessoas interessadas em criar e compartilhar suas narrativas pessoais que se configuram como um tipo de narrativa digital (Robin, 2008), talvez o mais utilizado para fins educacionais. Esse tipo de narrativa combina a arte de contar histórias com uma variedade de mídias (Robin, 2005; 2008). Aplicativos da Web 2.0, como o Flash, o MovieMaker, software para produção de blogs, entre outros, ou até mesmo aplicativos convencionais para produção de apresentação, como o PowerPoint, também podem ser utilizados para a produção de narrativas. A facilidade de manipulação dessas diferentes mídias permite que as pessoas sejam autoras, produtoras e disseminadoras de conhecimento. É notável, por exemplo, o uso de blogs como meio de disseminação de conhecimento, seja na forma textual, imagética ou animada (Almeida \& Valente, 2012).

Na educação, a narrativa digital reúne diversos objetivos, dentre os quais estimular a reflexão, promover a criatividade, o envolvimento dos alunos em atividades colaborativas e seu protagonismo no processo de ensino-aprendizagem (Barret, 2006). Nesse sentido, entende-se a narrativa digital como uma importante aliada para o ensino em diferentes áreas (Valente \& Almeida, 2014). Para esses autores, as narrativas podem ser utilizadas tanto para investigar o conhecimento que as pessoas expressam quanto para auxiliar processos de construção de conhecimento. Com a produção de narrativas de experiências com o auxílio dos recursos digitais, novas formas de produção de saber e práticas culturais de leitura e escrita são criadas, o que requer a urgente revisão dos processos de construção de conhecimento (Valente \& Almeida, 2014). Dessa forma, o estudo da narrativa digital tem chamado a atenção devido às competências que estão agregadas ao seu uso em contextos educacionais, estimulando pesquisas e aplicações práticas que ajudem a construir conhecimento sobre o tema.

Narrativa e experiência são, portanto, dois conceitos que se relacionam, pois o ato de narrar implica revelar histórias, vivências e organizá-las de modo que elas façam sentido e consigam transmitir uma informação. Logo, a narrativa produzida pelo aluno sobre a sua experiência no processo de ensino-aprendizagem se configura pelas suas singularidades, pelas relações que se constroem em cada vivência e que ajudam o sujeito a significar, por exemplo, aspectos da teoria na prática.

No ensino superior em Saúde ainda existe forte tendência por uma educação centralizada em conteúdos, distanciada da realidade de aprendizado do aluno, no qual o modelo hospitalar e orientado pela doença determina esse processo de formação profissional (González \& Almeida, 2010; Ceccim \& Feuerwerker, 2004). Esse tipo de formação não prepara os profissionais para trabalhar as dimensões subjetivas, sociais e culturais envolvidas no processo de adoecimento e tratamento dos indivíduos (Ribeiro, 2005). No contexto de formação em Atenção Primária à Saúde (APS) se percebe uma dificuldade de distanciamento desse modelo de ensino, sobretudo por suas raízes tão hegemônicas. A APS traz uma proposta de reorientação assistencial e, consequentemente, re- 
quer novos olhares para o ensino da Saúde. A atuação nesse nível do sistema busca uma formação mais integral e humana (Istúriz, 2015), na qual os estudantes sejam capazes de conhecer como as situações de um contexto amplo (social, familiar, demográfico) podem influenciar no processo saúde-doença (Carácio et al., 2014).

Nessa perspectiva, a narrativa digital se distancia de modelos de ensino de cunho prescritivo e verticalizado, como em geral acontece no ensino da Saúde, uma vez que o ato de narrar oferece espaço para o aluno se manifestar e assim poder participar ativamente do processo educativo, dialogando e compartilhando suas experiências.

A produção e a apresentação da narrativa digital envolvem diferentes linguagens e recursos midiáticos. Isso implica um olhar para a sua concepção, com o objetivo de entender como esses elementos são usados, que caminhos os sujeitos percorrem para representar a sua experiência e, com isso, criar condições para o seu aprendizado. Não há, portanto, um único modelo para analisar a narrativa digital, mas caminhos vindos de diferentes áreas. Um exemplo disso é o trabalho de Nora Paul (2012), que construiu uma taxonomia para as narrativas digitais. Segundo a autora, há cinco elementos específicos da narrativa digital: mídia, ação, relacionamento, contexto e comunicação.

Elemento 1: Mídia - O primeiro elemento diz respeito à combinação de mídias (textos, fotos, gráficos, áudio, vídeo etc.) usadas por quem desenvolve o conteúdo. Para esse elemento, são considerados quatro subelementos: configuração, tipo, fluxo e tempo/espaço. Configuração - são possíveis três diferentes configurações desses tipos de mídia: Conteúdo de mídia individual - a narrativa é desenvolvida somente por uma mídia (texto, vídeo ou áudio são os mais comuns); Conteúdo de mídia múltipla - inclui dois ou mais tipos de mídia na narrativa, mas esses elementos não estão interligados; e Narrativa em multimídia - utilizam dois ou mais tipos de mídia (por exemplo, imagens e texto) interligados na narrativa. Tipo - refere-se ao tipo de mídia que está sendo usada na construção da narrativa, como texto, gráfico, foto, vídeo, gráfico em movimento, áudio etc. Fluxo - identifica se o tipo de mídia é ao vivo ou gravado. Pode ser síncrono ou assíncrono. Tempo/Espaço - refere-se ao grau de alteração ou edição que o conteúdo pode sofrer, se foi editado ou se está sendo apresentado em tempo real.

Elemento 2: Ação - Envolve dois aspectos distintos do desenho da narrativa digital, o movimento do próprio conteúdo e a ação requerida pelo usuário para acessar o conteúdo. Em relação à ação, o conteúdo pode ser dinâmico (se ele se mover) ou estático (se não se mover). Se o leitor precisar fazer algo para o conteúdo se mover, ele é ativo; se não, é passivo.

Elemento 3: Relacionamento - Relaciona-se a versatilidade da narrativa digital. Caso seja possível interagir com o conteúdo, sem ficar limitado a ler/assistir/ouvir a história, ele é aberto; caso contrário, o conteúdo é fechado.

Elemento 4: Contexto - Refere-se àquilo que circunda e dá sentido a um tipo de produção. No caso das narrativas digitais, isso envolve as relações com outros materiais externos à narrativa, como o uso de links. Algumas questões determinarão o elemento "contextual" e a forma de sua operacionalização: os outros materiais são relacionados ou relevantes para a narrativa apresentada? Se são, a narrativa é hipermidiática, com uso de links; se não, ela é autoexplicativa. Os links aos materiais paralelos estão colocados dentro da narrativa? Se estiverem, os links são paralelos. Os links são dirigidos para materiais criados ou mantidos pelo site que apresenta a narrativa? Se forem, são links internos; se não, são externos. Pode também haver uma combinação de links internos e externos. Os links trazem material diferente do que já está exposto? Se sim, eles são suplementares; se não, eles são duplicativos. Pode haver um misto de links suplementares e duplicativos. Qual é o objetivo do link? Se for fornecer material específico para a narrativa, é um link contextual. Se for fornecer material similar ao tópico narrativo, é um link relacionado. 
Elemento 5: Comunicação - Relaciona-se à habilidade de se conectar com os outros por meio da mídia digital. Os aspectos da comunicação são: Configuração: um-a-um, um-para-vários, vários-para-um, muitos-para-muitos; Tipo: refere-se ao modo de comunicação estabelecido (bate-papo em tempo real, mensagens para fórum etc.); Direcionamento: a comunicação pode ser ao vivo, como os bate-papos; ou gravada, como nos fóruns; Moderação: refere-se ao grau pelo qual a comunicação é examinada pelos produtores do website; Objetivo: há três razões para que um link de comunicação seja estabelecido por um website de notícias: troca de informações, registro e comércio.

Essa taxonomia apresentada por Paul (2012) ajuda a entender como a narrativa digital é estruturada sob o ponto de vista das linguagens e recursos disponíveis pelas TDIC. E ela pode ser adaptada a diferentes tipos de produções, a partir do tipo de narrativa produzida. Desse modo, o objetivo do artigo é analisar narrativas digitais produzidas em um blog quanto às linguagens midiáticas utilizadas de acordo com alguns elementos da taxonomia de Paul (2012).

\section{Materiais e Métodos}

Trata-se de uma pesquisa qualitativa descritiva, cujo contexto é a disciplina de Atenção Integral à Saúde (AIS), que integra o currículo do curso de graduação em Medicina de uma universidade pública brasileira. Essa disciplina objetiva a formação em APS e representa o primeiro contato do aluno com o paciente/usuário do serviço de saúde no seu contexto social e cultural. Nesse processo de formação, o estudante precisa aprender a lidar com as questões que envolvem o indivíduo de forma holística e não apenas olhando para a doença. Por conseguinte, é necessário criar condições para que ele reflita sobre a sua experiência e construa conhecimento a partir desse diálogo entre a teoria e a prática, valorizando o que esta última oferece, como as singularidades relacionadas à experiência individual do paciente frente ao adoecimento ou à prevenção e promoção da saúde, uma vez que esta é a base que orienta a APS.

Os sujeitos do estudo são 18 alunos e uma professora da disciplina, que realizaram suas atividades práticas na Clínica da Família da Comunidade Santa Marta, Rio de Janeiro, no segundo semestre de 2014. Os alunos construíram, ao longo do semestre, narrativas digitais como atividades de reflexão sobre o contato com o paciente/usuário do serviço de saúde na comunidade e as perspectivas relacionadas a esse processo de formação em APS. Para a produção das narrativas digitais, criaram blogs no ambiente virtual de aprendizagem Vivências: experiências do processo de adoecimento e tratamento (http://ltc ead.nutes.ufrj. br/vivencias).

Para compor as análises deste artigo, escolheu-se o blog de um aluno da disciplina, caracterizado como Med10, a fim de analisar a forma como as narrativas foram construídas a partir da classificação proposta por Paul (2012). Cabe ressaltar que a pesquisa recebeu parecer favorável do Comitê de Ética e Pesquisa (CEP), sob o n652.119, de 09 de abril de 2014.

\section{Resultados e Discussão}

Esses resultados envolvem as análises da narrativa digital sob a perspectiva dos elementos que a constituíram, ou seja, identificou-se a forma como a narrativa foi construída, qual a mídia utilizada e como sua apresentação se configurou de acordo com a taxonomia apresentada por Paul (2012) e adaptada para este estudo. O elemento Mídia envolve a combinação de linguagens midiáticas (textos, fotos, gráficos, áudio, vídeo etc.) usadas por quem desenvolve o conteúdo da narrativa digital. Esse elemento, por sua vez, se subdivide em quatro subelementos: configuração, tipo, fluxo e tempo/espaço (Paul, 2012). No entanto, para a produção narrativa apresentada nesse estudo, dois subelementos foram considerados na análise das narrativas: configuração e tipo. Em relação à configuração da mídia utilizada, são possíveis 
três diferentes configurações: Conteúdo de mídia individual; Conteúdo de mídia múltipla; e Narrativa em Multimídia. O subelemento Tipo refere-se à mídia ou linguagem que está sendo usada na construção da narrativa, como texto, gráfico, foto, vídeo, gráfico em movimento, áudio etc.

O blog do aluno Med10, denominado "Momento Saúde", apresentou 22 narrativas digitais. Quanto ao tipo de mídia/linguagem, utilizou texto, imagens/fotos e vídeos; em relação à configuração da mídia, construiu narrativas dos três tipos: conteúdo de mídia individual; conteúdo de mídia múltipla e narrativa em multimídia. As narrativas com conteúdo de mídia individual eram compostas não apenas por textos; algumas apresentavam somente imagens/fotos. Aquelas que apresentaram conteúdo de mídia múltipla continham texto e imagem/foto, mas esta última não estava interligada ao sentido da narrativa. As demais se constituíram como narrativas em multimídia e foram produzidas a partir de texto e imagens/fotos ou texto e vídeos. A Figura 1 ilustra exemplos dos três tipos de configuração de mídia utilizados por Med10.

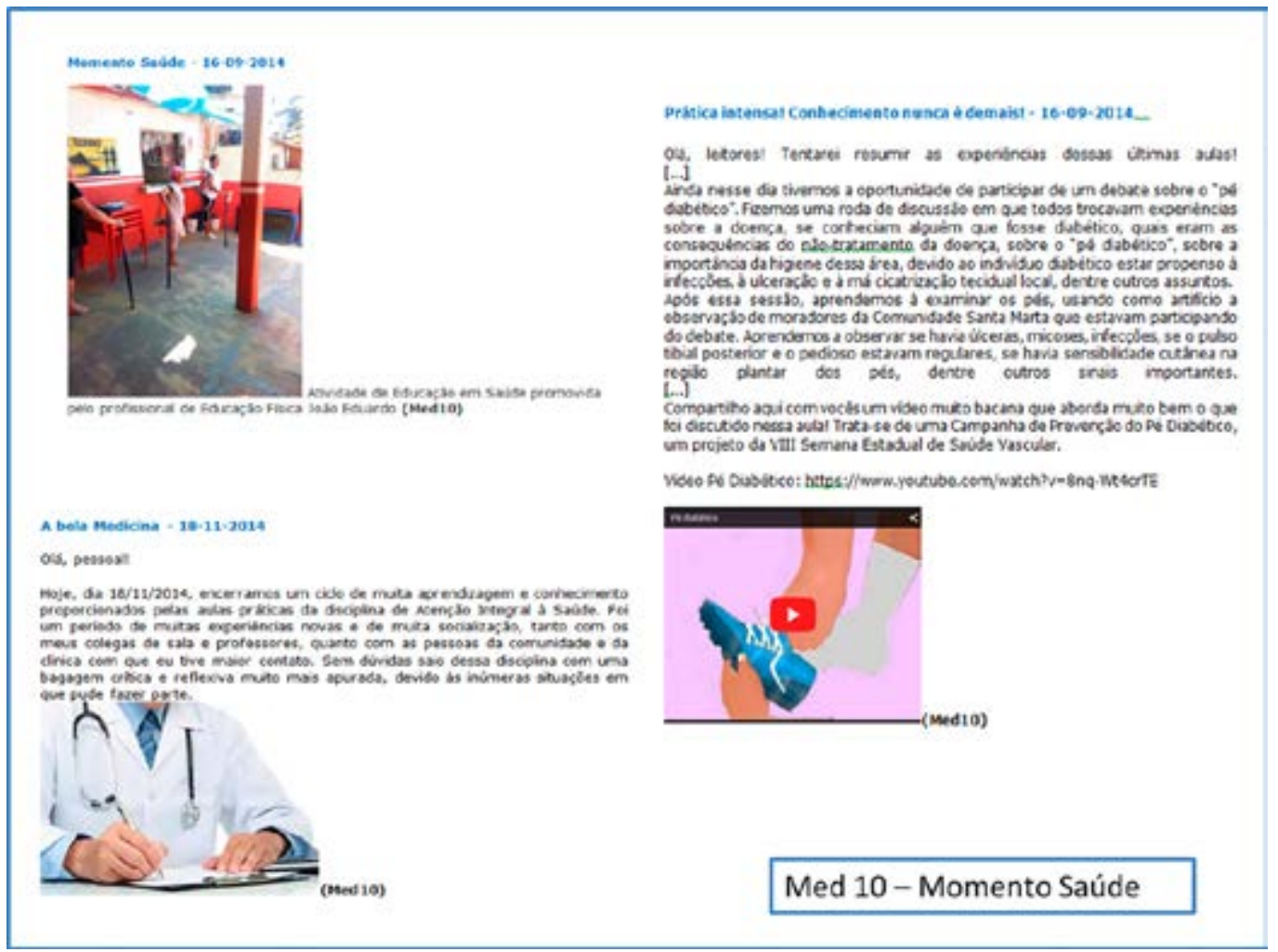

Figura 1: Exemplos de narrativas digitais com os três tipos de configuração de mídia.

Uma característica das narrativas de Med10 foi o uso de vídeos na produção de narrativas em multimídia (Figura 2). Esse tipo de linguagem midiática agregava à narrativa conhecimentos novos relacionados ao que o aluno havia aprendido na prática ou destacava algum tema relacionado à formação em APS, dessa maneira compartilhando com os demais suas experiências. Quando o aluno administrou sua primeira injeção, a vacina da hepatite $\mathrm{B}$, ele apresentou um vídeo que fala sobre a doença e os benefícios da vacinação. Na sua narrativa destacou: “Entusiasmado com o fato, procurei buscar mais informações sobre a doença e gostaria de fixá-las aqui! O Momento saúde de hoje foca na hepatite B" (Med10). Da mesma forma aconteceu a escolha dos outros vídeos, sempre relacionados ao contexto narrado, buscando potencializar aquela experiência a partir de diferentes formas, de modo que fizesse sentido na sua reflexão. Logo, o vídeo foi utilizado nas narrativas digitais com a finalidade de relacionar teoria e prática, apresentar novos conhecimentos sobre o aprendizado durante as vivências em AIS, sugerir temas de interesse aos outros alunos, ampliando assim, o alcance da narrativa. 


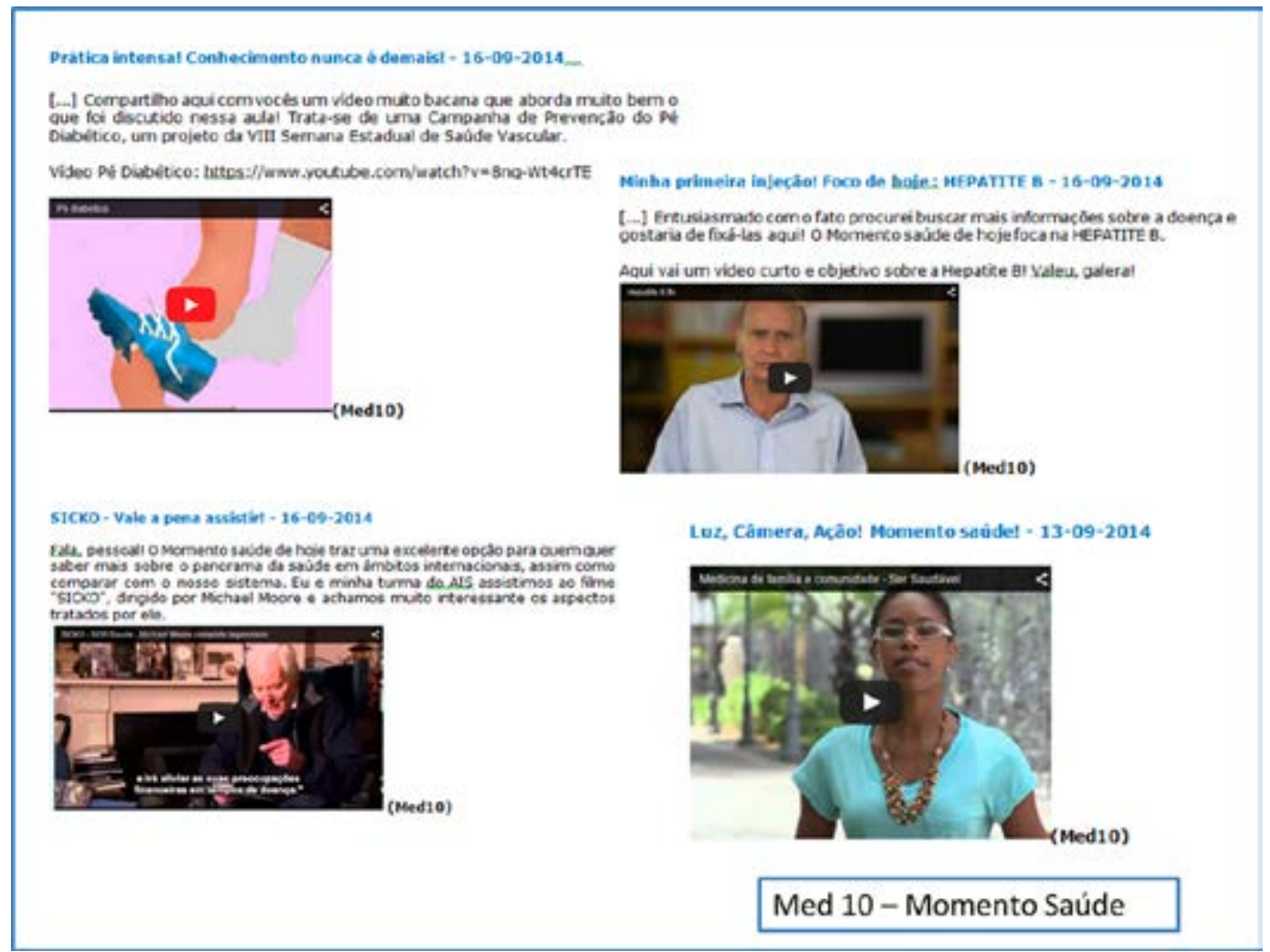

Figura 2: Exemplos de narrativas digitais que continham vídeos na sua composição.

Outra especificidade das narrativas apresentadas por Med10 foi o uso de imagens/fotos em produções que apresentavam apenas um tipo de mídia/linguagem (Figura 3). Nesses casos, por meio de fotografias ou imagens capturadas na internet, o aluno pode representar suas experiências de aprendizagem sem perder os significados atrelados a essas vivências. Nesse sentido, representou um importante caminho para um movimento reflexivo do aluno. Exemplo disso são as fotografias que guardam a particularidade de ser um instrumento de resgate para a memória, uma vez que nos leva de volta a um momento específico no tempo e ajuda na reflexão retrospectiva (JISC, 2012). 


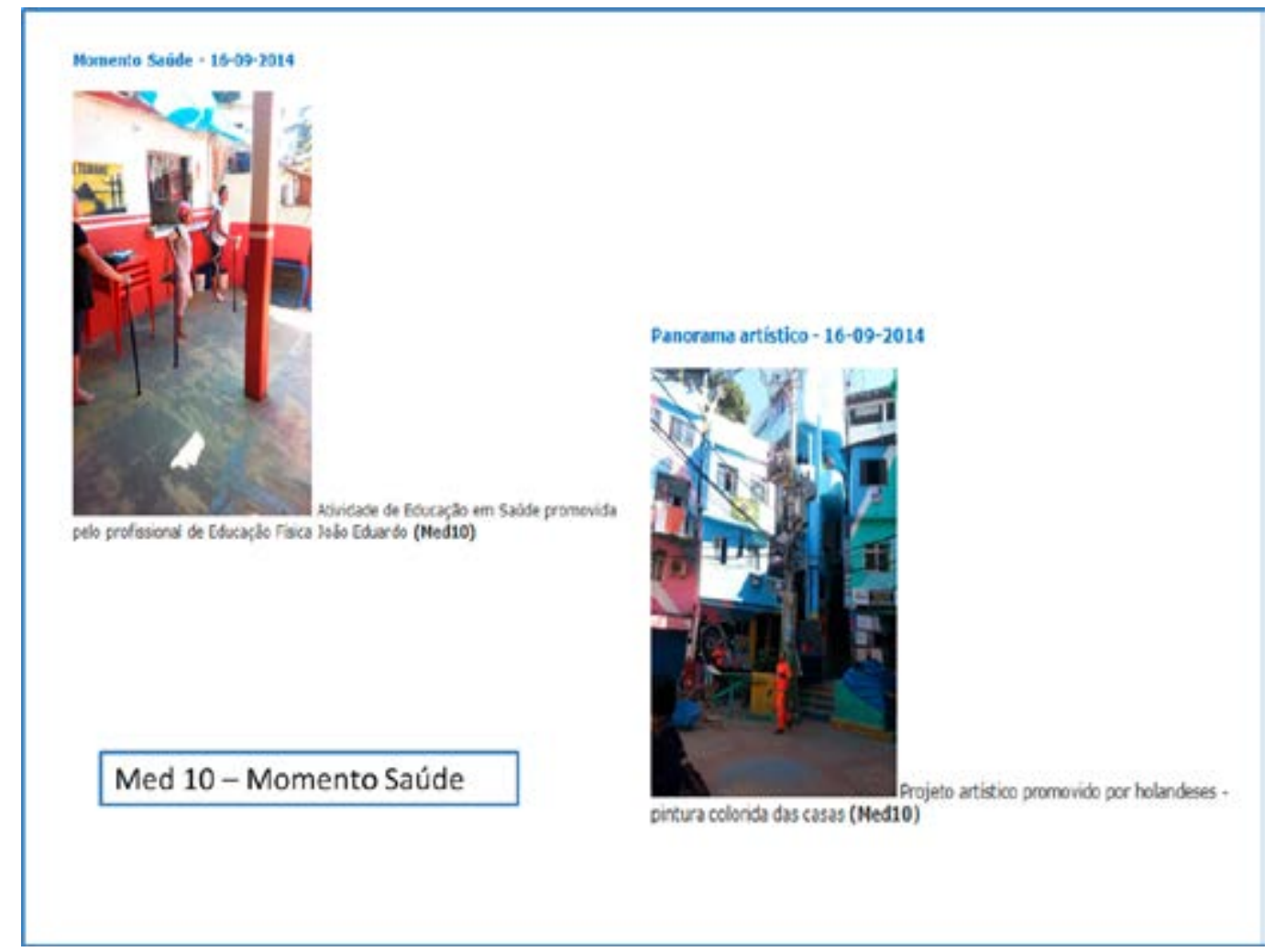

Figura 3: Exemplos de narrativas de conteúdo de mídia individual contendo imagens/fotos.

Os resultados encontrados demonstram o uso de diferentes linguagens na produção das narrativas, principalmente as imagens/fotos e os vídeos, e as três configurações descritas por Paul (2012), com destaque para as narrativas em multimídia. Isso corrobora outros estudos que também utilizaram essa perspectiva de análise. No trabalho de Bissolotti, Gonçalves \& Pereira (2015) no qual se analisou um hiperlivro, foram identificados dois tipos de configuração, narrativa em multimídia e conteúdo de mídia múltipla; em relação ao tipo de mídia, havia a presença de texto, imagem, vídeo e animação. Os autores também reconheceram que, pelo tipo de produção do estudo, não foi possível analisar as narrativas a partir de todos os elementos propostos por Paul (2012). No entanto, essa taxonomia apresentada pela autora não se limita ao seu contexto de construção, em ambientes online, podendo ser adaptada em outras produções. A narrativa digital representa, portanto, um novo tipo de abordagem que alcança a sua própria forma de expressão, principalmente pelas possibilidades oferecidas pelas tecnologias digitais (Bissolotti, Gonçalves \& Pereira, 2015).

A partir da análise da narrativa digital, sob esse enfoque pode-se considerar que o uso de diferentes linguagens pelo aluno para apresentar suas experiências em AIS e a forma como explorou o espaço do blog na produção das narrativas são aspectos que sugerem o reforço a determinadas habilidades e o interesse por trazer para o seu espaço educativo o que já utilizava fora dele. Conforme refere Gregori-Signes (2014), a produção das narrativas digitais está relacionada à maior autonomia do aluno e ao estímulo à sua iniciativa, reforçando a necessidade de aprender a aprender, refletir sobre as suas experiências e como representá-las da melhor forma para que veiculem significados para si e para os outros nesse contexto de formação profissional na área da saúde.

\section{Considerações Finais}

A análise do blog do aluno Med10 revelou a produção de narrativas digitais multimídia, em sua maioria, e o uso de outras linguagens midiáticas além do texto, como as imagens/fotos e os vídeos, sugerindo a 
compreensão do aluno a respeito do potencial reflexivo agregado a esse tipo de produção. Fazer uma foto de uma atividade e contar a sua experiência por meio dela é uma forma de agregar sentido e aprender a partir do olhar para o seu entorno, para a comunidade na qual o aluno estava inserido nesse processo de formação em APS. Da mesma forma, um diferencial nessas narrativas foi o modo como aluno utilizou o vídeo para complementar aquele momento de aprendizado, apresentando outra narrativa dentro da sua história, interligando conceitos e ampliando o alcance da sua experiência.

Os resultados deste artigo representam um olhar sobre a narrativa digital, uma vez que a sua discussão na educação e particularmente, no ensino da saúde, possui um alcance maior devido à sua relação com a experiência no aprendizado e a reflexão do aluno. Contudo, são questões que podem ser exploradas a partir do entendimento de como o aluno constrói a sua narrativa, conforme apresentado. Assim, as discussões tecidas permitem um entendimento sobre a narrativa digital no ato de educar como uma abordagem que pode ser trabalhada em uma perspectiva de mudança de práticas tradicionais de ensino, ajudando a reconfigurar o papel dos sujeitos educandos. Quando se discute o ensino em Saúde, propostas pedagógicas que invistam na integração das TDIC representam um caminho a ser explorado no sentido de dialogar com esse cenário de transformações na formação dos profissionais em APS.

\section{Agradecimentos}

À Fundação de Amparo à Pesquisa do Estado do Rio de Janeiro (Faperj) e ao Conselho Nacional de Desenvolvimento Científico e Tecnológico (CNPq).

\section{Referências Bibliográficas}

Almeida, M. E. B. \& Valente, J. A. (2012). Integração currículo e tecnologias e a produção de narrativas digitais. Currículo sem Fronteiras, 12(3), 57-82. Disponível em: http://www.curriculosemfronteiras.org/ vol12iss3articles/almeida-valente.pdf

Barret, H. C. (2006). Researching and Evaluating Digital Storytelling as a Deep Learning Tool. In Proceedings of Society for Information Technology \& Teacher Education International Conference (pp. 647-654).

Bissolotti, K., Gonçalves, B. \& Pereira, A. T. (2015). Hyperbook: analysis of digital narratives of creation and online edition of educational hypermedia. In $7^{\circ}$ Congresso Nacional de Ambientes Hipermídia para Aprendizagem. São Luís, MA.

Boase, C. (2013). Digital Storytelling for Reflection and Engagement: a study of the uses and potential of digital storytelling. Centre for Active Learning \& Department of Education, University of Gloucestershire. Disponível em: https://gjamissen.files.wordpress.com/2013/05/boase assessment.pdf

Bruner, J. S. (1996). Cultura da Educação. Lisboa: Edições 70.

Bruner, J. S. (1991). The narrative construction of reality. Critical Inquiry, 17, 1-21.

Carácio, F. C. C, Conterno, L. de O., Oliveira, M. A. de C., Oliveira, A. C. H. de, Marin, M. J. S. \& Braccialli, L. A. D. (2014). A experiência de uma instituição pública na formação do profissional de saúde para atuação em atenção primária. Ciência \& Saúde Coletiva, 19(7), 2.133-2.142. https://dx.doi.org/10.1590/141381232014197.08762013

Ceccim, R. B. \& Feuerwerker, L. C. M. (2004). Mudança na graduação das profissões de saúde sob o eixo da integralidade. Cadernos de Saúde Pública, 20(5), 1.400-1.410. https://dx.doi.org/10.1590/S0102$311 \times 2004000500036$ 
Gonzalez, A. D. \& Almeida, M. J. de. (2010). Integralidade da saúde: norteando mudanças na graduação dos novos profissionais. Ciência \& Saúde Coletiva, 15(3), 757-762. https://dx.doi.org/10.1590/S1413$\underline{81232010000300018}$

Gregori-Signes, C. (2014). Digital Storytelling and Multimodal Literacy in Education. Porta Linguarum, 22.

Istúriz, O. F. (2015). Avanzando hacia la nueva APS: cuidado integral de la salud con la participación de todas y todos. In L. Giovanella (Ed.). Atención primaria de salud en Suramérica. Rio de Janeiro: Isags/Unasur.

JISC. (2012). Digital approaches to academic reflection a digital storytelling study guide. Leeds Metropolitan University.

Paul, N. (2012). Elementos das narrativas digitais. In P. Ferrari (Ed.). Hipertexto e hipermídia: as novas ferramentas da comunicação digital. São Paulo: Contexto.

Ribeiro, V. M. B. (2005). Discutindo o conceito de inovação curricular na formação dos profissionais de saúde: o longo caminho para as transformações no ensino médico. Trabalho, Educação e Saúde, 3(1). https://dx.doi.org/10.1590/S1981-77462005000100006

Robin, B. (2005). The educational uses of digital storytelling. In University of Houston. Educational uses of digital storytelling website. Disponível em: http://digitalstorytelling.coe.uh.edu/

Robin, B. R. (2008). Digital storytelling: a powerful technology tool for the 21st century classroom. Theory Into Practice, 47(3), 220-228. Doi: 10.1080/00405840802153916

Valente, J. A. \& Almeida, M. E. B. (2014). Narrativas digitais e o estudo de contextos de aprendizagem. Em Rede, Revista de Educação a Distância, 1(1), 32-50. Disponível em: http://www.aunirede.org.br/revista/ index.php/emrede/article/view/10/22 